\author{
А. А. Блудов, Г. А. Горбатовский, В. С. Павлов, А. Ф. Суворов \\ AО «НПП "Радар ММС"» \\ ул. Новосельковская, д. 37, лит. А, Санкт-Петербург, 197375, Россия \\ | Оценка и компенсация поляризационных погрешностей
}

\begin{abstract}
Аннотация. Рассмотрено решение задачи компенсации поляризационных погрешностей пеленгации локационного объекта, осуществляемой методом конического сканирования, на уровне сигнальной обработки, что позволяет обойти поляризационные ограничения при проектировании антенн. Цель работы состоит в обосновании модели поляризационных погрешностей пеленгации методом конического сканирования и разработке алгоритмического способа коррекции данного метода относительно произвольных поляризационных условий радиолокационного взаимодействия. Результаты представлены аналитической моделью вместе с количественными оценками поляризационных погрешностей пеленгации, вычислительной прочедурой компенсации этих погрешностей, а также анализом факторов неидеальности предлагаемой компенсации, раскрывающим ее практическую применимость.
\end{abstract}

Ключевые слова: коническое сканирование, локационный сигнал, оценивание, пеленгация, поляризация, цифровая обработка сигналов

Для цитирования: Оценка и компенсация поляризационных погрешностей в пеленгационном методе конического сканирования / А. А. Блудов, Г. А. Горбатовский, В. С. Павлов, А. Ф. Суворов // Изв. вузов России. Радиоэлектроника. 2018. № 2. C. $47-54$.

A. A. Bludov, G. A. Gorbatovskij, V. S. Pavlov, A. F. Suvorov

JSC "Radar MMS"

37A, Novoselkovskaya Str., 197375, St. Petersburg, Russia

\title{
Estimation and Adjustment of Polarization Errors in Conical Scan Method
}

Abstract. The article proposes a solution of a problem of polarization error compensation for radar object direction finding by means of conical scan method. The solution is considered at signal processing level that makes possible to avoid polarization limitations in antennas engineering. The purpose of the article is to substantiate a model for polarization-induced errors by conical scan direction finding method and to develop an algorithmic technique for the considered method correction with regard to arbitrary polarization conditions of radar interaction. The results are presented by analytical model along with quantitative estimates of polarization-induced errors of direction finding and the computational procedure of the error compensation as well as by analysis of imperfectness factors for the proposed procedure exposing its practical applicability.

Key words: conical scan, radar signal, estimation, direction finding, polarization, digital signal processing

For citation: Bludov A. A., Gorbatovskij G. A., Pavlov V. S., Suvorov A. F. Estimation and Adjustment of Polarization Errors in Conical Scan Method. Izvestiya Vysshikh Uchebnykh Zavedenii Rossii. Radioelektronika [Journal of the Russian Universities. Radioelectronics]. 2018, no. 2, pp. 47-54. (In Russian)

Введение. Метод конического сканирования [1], [2], применяемый для двухплоскостной пеленгации локационного объекта (ЛО) уже несколько десятков лет, сохраняет свою актуальность и в настоящее время. Причина его востребованности для вновь создаваемых радиолокационных систем (РЛС) состоит в сведении всей пространственно-временной обработки к одному приемопередающему каналу, что позволяет ограничиться малыми аппаратными ресурсами при технической реализации данного метода.

Один из известных и существенных источников погрешностей рассматриваемого пеленгационного метода сопутствует ситуации, при которой 
коническое сканирование антенного луча сопровождается вращением плоскости поляризации используемой антенны, что приводит к зависимости показателей качества пеленгации от поляризационных характеристик сигнала, поступающего от ЛО. Если же коническое сканирование антенного луча организовано посредством вращения отражателя (зеркала антенны) при неподвижном облучателе либо используется антенна круговой поляризации, то указанный источник погрешностей отсутствует. Согласно этим соображениям обоснованы рекомендации [2] по практической реализации пеленгационного метода конического сканирования на уровне проектирования соответствующих приемопередающих антенн.

Однако нередко использование пеленгационного метода конического сканирования предпочтительно при линейной поляризации антенны как на излучение, так и на прием. Это может быть связано как с упрощением конструкции сканирующей антенны (что особенно важно для фазированных антенных решеток [3]), так и со спецификой применения пеленгационного метода конического сканирования. В качестве примера укажем возможную обусловленность конического сканирования антенного луча вращением платформы, на которой размещена сама РЛС [4].

Рассмотрение отмеченных обстоятельств в контексте возможностей, предоставляемых цифровой обработкой сигналов в современных РЛС [5], определяет задачу компенсации поляризационного источника погрешностей в пеленгационном методе конического сканирования на уровне вычислительной процедуры, что требует расширения поляризационной модели для данного метода.

Поляризационная модель локационного сигнала для пеленгационного метода конического сканирования. Согласно задаче исследования рассмотрим условия совместного синхронного вращения плоскости поляризации приемопередающей антенны и конического сканирования антенного луча с круговой частотой $\Omega=2 \pi / T$, где $T$ - период конического сканирования.

Основная функциональная зависимость, подлежащая анализу в проводимом исследовании, задается связью между эффективной площадью рассеяния $\sigma$ (ЭПР) ЛО и углом поворота плоскости поляризации приемопередающей антенны $\Omega t$ в момент времени $t$. Считая, что поляризационная матрица рассеяния ЛО [6] постоянна на периоде $T$, опишем временное изменение ЭПР на данном периоде функцией $\sigma(\Omega t)$.
Для реального ЛО о зависимости $\sigma(\Omega t)$ можно судить по экспериментальным данным, отражающим различие его ЭПР в горизонтальной $\sigma_{\mathrm{H}}$ и вертикальной $\sigma_{\mathrm{V}}$ плоскостях поляризации радиолокационного контакта с ЛО либо в двух других аналогичных ортогональных плоскостях [7]. Например, в [8] это различие составляет 0.4 дБ (примерно $10 \%$ ) по усредненным значениям величин $\sigma_{\mathrm{H}}$ и $\sigma_{\mathrm{V}}$, тогда как в других случаях оно может быть более ощутимым [7], а также использоваться в качестве информационного признака при решении некоординатных задач [9].

Принципиально важным свойством зависимости $\sigma(\Omega t)$ является ее повторяемость с периодом $\pi: \sigma(\Omega t)=\sigma(\Omega t \pm i \pi) \quad(i=0,1, \ldots)$, поскольку для ЭПР, являющейся энергетическим показателем, плоскость поляризации радиолокационного контакта с ЛО при ее повороте на угол $\pi$ остается прежней [7]. При рассматриваемом непрерывном вращении плоскости поляризации приемопередающей антенны поляризационное изменение ЭПР происходит на удвоенной частоте $2 \Omega$, следовательно, спектральное разложение процесса $\sigma(\Omega t)$ будет содержать только четные гармоники частоты $\Omega$.

Изменение ЭПР во времени можно также представить в виде модуляции амплитудного поляризационного коэффициента приема $K$ [10], при этом отмеченное выше свойство четности гармоник спектрального разложения относится и к временному процессу $K(\Omega t)$. В [11] показано, что для решения практических задач разложение коэффициента $K(\Omega t)$ в ряд Фурье тригонометрической формы [12] можно ограничить постоянной составляющей $P$ и второй гармоникой с амплитудой $p$ :

$$
\begin{aligned}
& K(\Omega t) \cong P+p \cos (2 \Omega t-2 \eta)= \\
& =P+p_{\mathrm{C}} \cos 2 \Omega t+p_{\mathrm{S}} \sin 2 \Omega t,
\end{aligned}
$$

где $p_{\mathrm{C}}=p \cos 2 \eta$ и $p_{\mathrm{S}}=p \sin 2 \eta-$ коэффициенты при косинусной и синусной составляющих второй гармоники; $2 \eta$ - начальная фаза второй гармоники, соответствующая углу плоскости поляризации радиолокационного контакта с ЛО, при котором ЭПР максимальна.

Коническое сканирование антенного луча вносит амплитудную модуляцию в низкочастотную огибающую $u(t)$ принимаемого локационного сигнала, причем глубина и фаза этой моду- 
ляции определяются сферическими координатами ЛО - соответственно широтой $\theta$ и долготой $\varphi$.

Углы $\theta$ и $\quad \varphi$ связаны с горизонтальной $\theta_{X}=\theta \cos \varphi$ и вертикальной $\theta_{Y}=\theta \sin \varphi$ угловыми координатами ЛО, отсчитываемыми в декартовой координатной системе $X Y Z$ (в плоскостях $X Z$ и $Y Z$ соответственно - согласно геометрической модели из [2]) от опорного направления $0 Z$, совпадающего с осью конического сканирования. Для дальнейших аналитических действий введем вектор $\boldsymbol{\theta}=\left(\begin{array}{ll}\theta_{X} & \theta_{Y}\end{array}\right)^{\mathrm{T}}=\theta\left(\begin{array}{ll}\cos \varphi & \sin \varphi\end{array}\right)^{\mathrm{T}} \quad$ двух угловых координат ЛО в координатной системе $X Y Z$, где "т" - знак транспонирования.

Процесс $u(t)$ представим в виде произведения амплитудного $A(t)$, поляризационного $K(\Omega t)$ и пространственного $G(t)$ сомножителей, последний из которых определяется диаграммой направленности антенны (ДНА) и является функцией времени ввиду конического сканирования антенного луча. Для упрощения анализа допустим, что время корреляции процесса $A(t)$, описывающего амплитудные флюктуации принимаемого локационного сигнала, значительно превышает период $T$, следовательно, модель процесса $u(t)$ на данном периоде можно представить в виде: $u(t)=A G(t) K(\Omega t)$, где $A=\mathrm{E}\{A(t)\} \quad$ - усредненный амплитудный сомножитель; $\mathrm{E}\{\cdot\}=(1 / T) \int_{-T / 2}^{T / 2}(\cdot) d t$ - оператор усреднения на периоде $T$.

В силу периодичности изменения углового положения ДНА во времени и по аналогии с [1] процесс $G(t)$ удобно выразить через усеченный до $N$ гармоник ряд Фурье тригонометрической формы [12]:

$$
G(t) \cong \sum_{n=0}^{N} g_{n}(\theta) \cos (n \Omega t-n \varphi),
$$

где $g_{n}(\theta), n=\overline{0, N}-n$-й коэффициент разложения.

Существенной для проводимого исследования является возможность исключения низкоамплитудных гармоник из (2). С этой целью определим удельный вес $w_{n}(\theta)$ для $n$-й гармоники $(n=\overline{1, N})$ через отношение ее амплитуды к постоянной составляющей $(n=0): w_{n}(\theta)=g_{n}(\theta) / g_{0}(\theta)$. Количественную оценку этого отношения выполним на основе двумерной гауссовской аппроксимации ДНА из [1], вводя обозначения: $\theta_{0}-$ смещение оси антенного луча относительно опорного направления; $g_{0}(0)$ - значение ДНА при $\theta=0$. Тогда: $w_{n}(\theta)=2 I_{n}(\mu \theta) / I_{0}(\mu \theta)$, где $I_{n}(\cdot)-$ модифицированная функция Бесселя $n$-го порядка [13]; $\mu=-2 \ln g_{0}(0) / \theta_{0}-$ метрический показатель ДНА для метода конического сканирования.

Зависимости коэффициентов $w_{n}(\theta)$ при $n=1-4$ от нормированной угловой координаты $\theta / \theta_{0}$ показаны на рис. 1 для одного из типовых значений $g_{0}(0)=0.6[14]$ (другим значениям $g_{0}(0)$ соответствуют кривые с незначительными отличиями от приведенных на рис. 1). Видно, что в пределах линейной рабочей области пеленгации при $\theta<0.6 \theta_{0}$ коэффициенты $w_{3}(\theta)$ и $w_{4}(\theta)$ малы (менее 0.01), поэтому в (2) можно ограничиться значением $N=2$, считая коэффициент $w_{n}(\theta)$ быстро убывающим при увеличении $n$.

Если предположить, что разложение (1) расширено за счет высших гармоник с частотами $4 \Omega, 6 \Omega, \ldots$, то мультипликативные комбинации этих гармоник, содержащие составляющие с частотой $\Omega$, могут быть образованы только вместе с гармониками разложения (2), которые имеют частоты, превышающие $2 \Omega$. Удельный вес таких гармоник (начиная с частоты $3 \Omega$ ) в разложении (2) пренебрежимо мал (рис. 1), следовательно, указанные мультипликативные комбинации можно исключить из последующих аналитических действий. Таким образом, поляризационная модель (1) является достаточно точной для проводимого исследования, поскольку увеличение числа гармоник, входящих в эту модель, не влечет за собой заметного эффекта.

Выделение угловой координатной информации, поляризационные погрешности пеленгации. Обработку принимаемого локационного сигнала, основывающуюся на демодуляции его низкочастотной огибающей [1], [2], рассмотрим расширенно, используя полигармоническую мо-

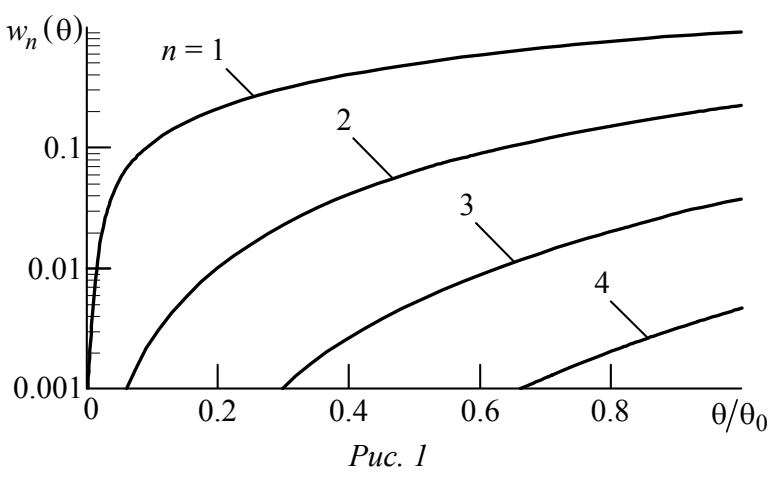


дель процесса $u(t)$ на периоде $T$. Раскрывая в данной модели произведение сумм (1) и (2), определим для процесса $u(t)$ усредненную величину $U=\mathrm{E}\{u(t)\}$ и двухэлементный вектор коэффициентов при косинусной $c_{n}$ и синусной $s_{n}$ составляющих $n$-й гармоники разложения в ряд Фурье тригонометрической формы [12]: $\left(\begin{array}{ll}c_{n} & s_{n}\end{array}\right)^{\mathrm{T}}=2 \mathrm{E}\left\{u(t)(\cos n \Omega t \quad \sin n \Omega t)^{\mathrm{T}}\right\}$.

Согласно обоснованному ранее значению $N=2$ в модели процесса $u(t)$ не будем учитывать гармоники его разложения с частотами, превышающими $2 \Omega$. Тогда, полагая $n=1,2$ и проводя последовательность аналитических действий на основе (1) и (2), получим:

$$
\begin{gathered}
U \cong A P g_{0}(\theta) \\
\left(\begin{array}{c}
c_{1} \\
s_{1}
\end{array}\right) \cong A g_{1}(\theta)\left(\begin{array}{cc}
P+p_{\mathrm{C}} / 2 & p_{\mathrm{S}} / 2 \\
p_{\mathrm{S}} / 2 & P-p_{\mathrm{C}} / 2
\end{array}\right)\left(\begin{array}{c}
\cos \varphi \\
\sin \varphi
\end{array}\right), \\
\left(\begin{array}{c}
c_{2} \\
s_{2}
\end{array}\right) \cong A g_{0}(\theta)\left(\begin{array}{c}
p_{\mathrm{C}} \\
p_{\mathrm{S}}
\end{array}\right)+A P g_{2}(\theta)\left(\begin{array}{c}
\cos 2 \varphi \\
\sin 2 \varphi
\end{array}\right) .
\end{gathered}
$$

Принятое ранее допущение относительно медленного характера амплитудных флюктуаций принимаемого локационного сигнала позволяет описать вычислительную процедуру пеленгационного метода конического сканирования исходя из (3) и (4) в виде: $\mathbf{y}=\left(\begin{array}{ll}c_{1} & s_{1}\end{array}\right)^{\mathrm{T}} /(\mu U)$, где $\mathbf{y}=\left(\begin{array}{ll}y_{X} & y_{Y}\end{array}\right)^{\mathrm{T}}=y(\cos \psi \quad \sin \psi)^{\mathrm{T}}-$ вектор, имеющий длину $y$, угол $\psi$, элементы $y_{X}$ и $y_{Y}$ которого являются оценками соответствующих угловых координат ЛО $\theta_{X}$ и $\theta_{Y}$.

В линейной рабочей области пеленгации преобразование вектора $\boldsymbol{\theta}$ в вектор у при отсутствии поляризационной модуляции осуществляется с единичным коэффициентом $\left(w_{1}(\theta) \cong \mu \theta\right)$, а связь векторов у и $\boldsymbol{\theta}$ может быть выражена через векторно-матричную форму:

$$
\mathbf{y}=(I+Q) \boldsymbol{\theta},
$$

где $I$ - единичная матрица размеров $2 \times 2$;

$$
Q=\frac{1}{2 P}\left(\begin{array}{cc}
p_{\mathrm{C}} & p_{\mathrm{S}} \\
p_{\mathrm{S}} & -p_{\mathrm{C}}
\end{array}\right)=q\left(\begin{array}{cc}
\cos 2 \eta & \sin 2 \eta \\
\sin 2 \eta & -\cos 2 \eta
\end{array}\right)
$$

- матрица координатного преобразования, обусловленного поляризационной модуляцией; $q=p /(2 P)$ - нормированный амплитудный коэффициент второй гармоники поляризационной модели (1).
Из (6) видно, что формируемую векторную оценку можно представить как сумму $\mathbf{y}=\boldsymbol{\theta}+\boldsymbol{\Delta}$, в которой $\boldsymbol{\Delta}=Q \boldsymbol{\theta}=\Delta(\cos \xi \quad \sin \xi)^{\mathrm{T}}-$ вектор из двух погрешностей пеленгации ЛО (в плоскостях $X Z$ и $Y Z$ соответственно), вызванных поляризационной модуляцией; $\Delta=q \theta$ и $\xi=2 \eta-\varphi-$ длина и угол вектора $\boldsymbol{\Delta}$. Иллюстрация трех векторов $\boldsymbol{\theta}, \mathbf{y}, \boldsymbol{\Delta}$ приведена на рис. 2 относительно горизонтальной плоскости пеленгации, представленной осью $0 X$, а для каждого из этих векторов указаны соответствующие ему длина $\theta, y, \Delta$ и угол $\varphi, \psi, \xi$.

Различие истинной $\varphi$ и оценочной $\psi$ долготы ЛО можно трактовать как поворот измерительной координатной системы $X Y Z$ вокруг оси $0 Z$ на угол $\chi=\varphi-\psi$ (рис. 2). Этот угол найдем из уравнения треугольника, образованного тремя изображенными векторами, и запишем:

$$
\chi=\operatorname{arctg}\left(\frac{q \sin \gamma}{1+q \cos \gamma}\right)
$$

где $\gamma=\varphi-\xi=2 \varphi-2 \eta-$ удвоенная разность долготы ЛО и угла поляризации его сигнала.

Зависимости угла $\chi$ от разности $\gamma$ изображены на рис. 3 для двух случаев: $a$ ) наибольшей глубины поляризационной модуляции при $p / P=1 \quad(q=1 / 2)$; б) пассивной пеленгации ЛО, являющегося излучающим диполем [2], что соответствует отношению $p / P=2 / 3(q=1 / 3)$.

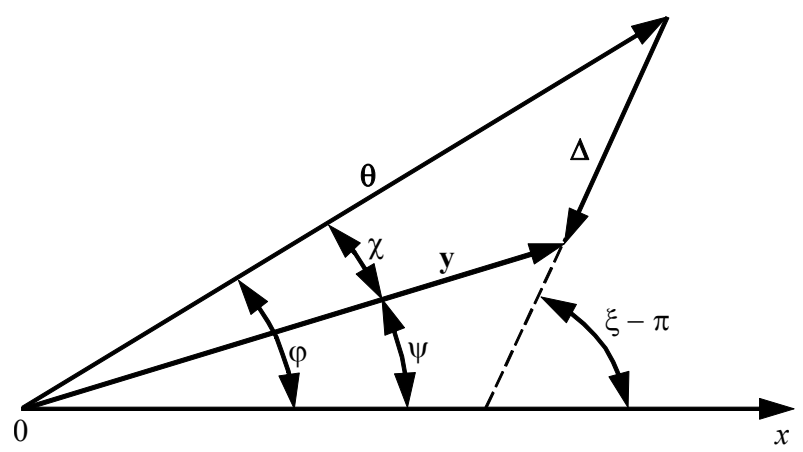

Puc. 2

Анализ (8) и рис. 3 показывают близкий к гармоническому характер зависимости $\chi$ от $\gamma$, при этом экстремумы данной зависимости имеют равные абсолютные значения и незначительно удаляются от оси ординат с увеличением $q$. Связь координаты первого максимума $\chi_{\mathrm{m}}$ при $\gamma>0$ с параметром $q$ в данной зависимости найдем исходя из равенства нулю производной от (8) по $\gamma: \chi_{\mathrm{m}}=\arcsin q \cong q$. 


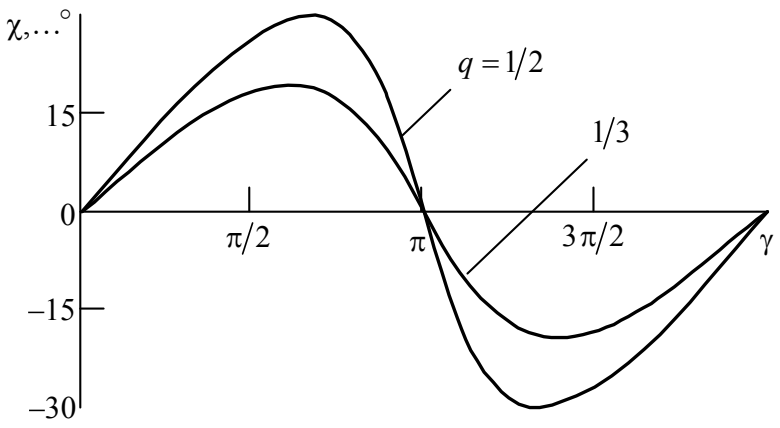

Puc. 3

При этом указанным ранее случаям $a$ ) и б) соответствуют значения $\chi_{\mathrm{m}} \cong 30^{\circ}$ и $\chi_{\mathrm{m}} \cong 19^{\circ}$.

Искажение масштаба формируемых оценок угловых координат ЛО, возникающее ввиду поляризационной модуляции, определим на основе рис. 2 через отношение $y / \theta \cong 1+q \cos \gamma$. Это отношение имеет периодичность по $\gamma$, а его максимальное и минимальное значения различаются между собой в $(1+q) /(1-q)$ раз, что для двух указанных ранее случаев $а$ ) и б) составляет: в 3 раза - при $q=1 / 2$ и в 2 раза - при $q=1 / 3$.

Полученные частные количественные значения поляризационных погрешностей для случая б) совпадают с результатами из [2], где они характеризуются как крайне негативные и способные привести к сбою в системе управления, использующей данные об угловых координатах ЛО. Следовательно, поляризационные погрешности играют весомую негативную роль при практическом применении пеленгационного метода конического сканирования и требуют принятия мер по их компенсации.

Изложенная методика анализа поляризационных погрешностей пеленгационного метода конического сканирования не только учитывает различие зависимостей $\sigma(\Omega t)$ для реальных ЛО, но и раскрывает связь параметров поляризации локационного сигнала с погрешностями пеленгации в векторно-матричном виде, что может служить исходной моделью для компенсации данных погрешностей.

Вычислительная процедура компенсации поляризационных погрешностей пеленгации. Очевидный способ рассматриваемой компенсации непосредственно следует из (6) и (7) и состоит в умножении формируемого вектора у на матрицу, обратную матрице $(I+Q)$. Эту обратную матрицу получим после аналитических действий: $(I+Q)^{-1}=(I-Q) /\left(1-q^{2}\right)$.
Отметим, что матрица $Q$ зависит от неизвестных параметров поляризации локационного сигнала и требует самостоятельной оценки, которую, как видно из (5), нетрудно осуществить, используя первое слагаемое данного выражения. С этой целью введем матрицу $B$ с элементами, являющимися оценками соответствующих элементов матрицы $Q$, и величину $a$ в качестве оценки сомножителя $1 /\left(1-q^{2}\right)$, определяя их следующим образом:

$$
B=\frac{1}{2 U}\left(\begin{array}{cc}
c_{2} & s_{2} \\
s_{2} & -c_{2}
\end{array}\right) ; \frac{1}{a}=1-\frac{c_{2}^{2}+s_{2}^{2}}{4 U^{2}} .
$$

С учетом изложенного искомая процедура формирования вектора $\quad \mathbf{z}=\left(\begin{array}{ll}z_{X} & z_{Y}\end{array}\right)^{\mathrm{T}}=z\left(\begin{array}{ll}\cos \zeta & \sin \zeta\end{array}\right)^{\mathrm{T}}$ двух скорректированных относительно поляризационной модуляции оценок $z_{X}$ и $z_{Y}$ соответствующих угловых координат ЛО $\theta_{X}$ и $\theta_{Y}$ примет вид

$$
\mathbf{z}=a(I-B) \mathbf{y}
$$

где $a(I-B)$ - матрица компенсационного координатного преобразования, устраняющего поляризационные погрешности пеленгации; $z$ и $\zeta-$ длина и угол вектора $\mathbf{z}$.

Отметим, что вычисляемые по (9) оценки $B$ и $a$ являются смещенными ввиду наличия в (5) дополнительной составляющей, которая не связана с параметрами поляризации локационного сигнала. Учитывая это обстоятельство и приближенный характер (5), представим матрицу $B$ как сумму: $B=Q+D$, где $D$ - матрица смещений размеров $2 \times 2$, в которой первый $d_{11}$ и второй $d_{22}$ диагональные элементы определяются приближенным равенством $d_{11}=-d_{22} \cong d \cos 2 \varphi$, а междиагональные элементы $d_{12}$ и $d_{21}$ совпадают между собой и приближенно равны $d_{12}=d_{21} \cong d \sin 2 \varphi$, где $d=w_{2}(\theta) / 2$. Наличие смещения в оценке $a$ аналогичным образом следует из (5), а для самой этой оценки после несложных аналитических действий имеем: $1 / a \cong$ $\cong 1-q^{2}-d^{2}-2 q d \cos \gamma$, где угол $\gamma$ описан в (8).

С целью определения методических погрешностей компенсации, проводимой согласно (10), раскроем в этом выражении вектор у в соответствии с (6): $\mathbf{z}=a(I-B)(I+Q) \boldsymbol{\theta}$ и преобразуем матричное произведение: $(I-B)(I+Q)=I-Q^{2}-D-D Q$. Нетрудно показать, что $Q^{2}=q^{2} I$ и $D \boldsymbol{\theta} \cong d \boldsymbol{\theta}$. Та- 
ким образом, вектор $\mathbf{z}$ можно представить в виде: $\mathbf{z}=\alpha \boldsymbol{\theta}+\boldsymbol{\delta}$, где $\alpha=a\left(1-q^{2}-d\right)-$ мультипликативная компонента различия векторов $\mathbf{z}$ и $\theta$; $\boldsymbol{\delta}=-a D Q \boldsymbol{\theta}=\delta(\cos v \quad \sin v)^{\mathrm{T}}-$ векторная аддитивная компонента данного различия; $\delta=a q d \theta$ и $v=3 \varphi-2 \eta+\pi-$ длина и угол вектора $\delta$.

Негативный вклад компоненты $\delta$ в вектор z рассмотрим по аналогии с описанным ранее вкладом компоненты $\Delta$ в вектор $\mathbf{y}$. Аналогом угла $\chi$ будет угол $\varepsilon=\varphi-\zeta$, характеризующий эквивалентный поворот измерительной координатной системы $X Y Z$ вокруг оси $0 Z$ после компенсации поляризационных погрешностей пеленгации, а аналогами величин $y$ и $\Delta$ - соответствующие отношения $z / \alpha$ и $\delta / \alpha$, учитывающие сомножитель $\alpha$ в выражении для z. Тогда, принимая во внимание малость угла $\varepsilon$ и используя (8), нетрудно получить упрощенное выражение: $\varepsilon \cong($ aqd $/ \alpha) \sin \gamma$, где угол $\gamma$ определен ранее.

Наиболее важным является количественное выражение угла $\varepsilon$, характеризующее эффективность процедуры компенсации (10). Нетрудно видеть, что максимальное значение угла $\varepsilon$ равно $\varepsilon_{\mathrm{m}} \cong a q d / \alpha$, следовательно, угол $\varepsilon_{\mathrm{m}}$ меньше угла $\chi_{\mathrm{m}}$ примерно в $\alpha /(a d) \cong$ $\cong 2\left(1-q^{2}-w_{2}(\theta) / 2\right) / w_{2}(\theta)$ раз, а о численном значении этого уменьшения можно судить по кривой $w_{2}(\theta)$ на рис. 1. Например, на границе линейной рабочей области пеленгации при $w_{2}\left(0.6 \theta_{0}\right) \cong 0.1$ и $q=1 / 2$ угол $\varepsilon_{\mathrm{m}}$ примерно в 15 раз меньше угла $\chi_{\mathrm{m}}$. Таким образом, в пределах линейной рабочей области пеленгации даже при наибольшем значении $q=1 / 2$ угол $\varepsilon_{\mathrm{m}}$ не превышает $2^{\circ}$, что пренебрежимо мало для практики.

Искажение масштаба вектора оценок $\mathbf{z}$ определим через отношение $z / \theta \cong \alpha-a q d \cos \gamma$ по аналогии с рассмотренным ранее отношением $y / \theta$. Из последнего выражения следует, что отношение $z / \theta$ имеет наибольшие отклонения от единицы при $q=1 / 2$, однако эти отклонения пренебрежимо малы при небольших углах $\theta<0.3 \theta_{0}$ и только лишь при приближении к границе линейной рабочей области пеленгации они становятся ощутимыми. При этом максимальное и минимальное значения, характеризующие разброс масштаба скорректированных оценок угловых координат ЛО, различаются между собой в $(\alpha+a q d) /(\alpha-a q d)$ раз, а на границе линейной рабочей области пеленгации это различие составляет не более $6 \%$, что приемлемо для практического применения процедуры (10).

Выводы. Одним из источников погрешностей рассмотренного пеленгационного метода в условиях вращения плоскости поляризации приемопередающей антенны синхронно с коническим сканированием антенного луча является поляризационная модуляция локационного сигнала, которая влечет за собой искажения ортогональности и масштаба оценок угловых координат ЛО в двух плоскостях пеленгации. Эти искажения проявляются тем сильнее, чем больше изменяется ЭПР при вращении плоскости поляризации радиолокационного контакта с ЛО. При этом периодическая составляющая изменения ЭПР во времени с достаточной для практики точностью может быть аппроксимирована только одной второй гармоникой частоты конического сканирования. Компенсация искажений оценок угловых координат ЛО основывается на векторно-матричной аналитической модели их формирования и состоит в коррекции вектора данных оценок за счет умножения на матрицу, в качестве элементов которой используются оценки параметров поляризации локационного сигнала, выделяемые по второй гармонике частоты конического сканирования. Методические погрешности, характеризующие неидеальность такой компенсации, проявляют себя лишь на границе линейной рабочей области пеленгации, а их количественное выражение несущественно для практического применения описываемой процедуры, реализующей компенсацию поляризационных погрешностей пеленгации.

\section{СПИСОК ЛИТЕРАТУРЫ}

1. Сколник М. Введение в технику радиолокационных систем. М.: Мир, 1965. 748 с.

2. Гуткин Л. С. Принципы радиоуправления беспилотными объектами. М.: Сов. радио, 1959. 384 с.

3. Инденбом М. В. Антенные решетки подвижных обзорных РЛС. Теория, расчет, конструкции. М: Радиотехника, 2015. 416 с.
4. Метод двухплоскостной широкоугольной пеленгации источника радиоизлучения / А. А. Блудов, Г. А. Горбатовский, В. С. Павлов, А. Ф. Суворов // Актуальные проблемы защиты и безопасности: тр. XIX Всерос. науч.практ. конф. / НПО СМ-РАРАН. СПб., 2016. Т. 4. С. 468-472.

5. Кузьмин С. З. Цифровая радиолокация. Введение в теорию. Киев: КВІЦ, 2000. 428 с. 
6. Козлов А. И., Логвин А. И, Сарычев В. А. Поляризация радиоволн: в 3 Кн. Кн. 2. Радиолокационная поляриметрия. М.: Радиотехника, 2007. 640 с.

7. Канарейкин Д. Б., Потехин В. А., Шишкин И. Ф. Морская поляриметрия. Л.: Судостроение, 1968. 328 с.

8. A Variability Study of Ka-Band HRR Polarimetric Signatures on Eleven T-72 Tanks. URL: https://www.researchgate.net/publication/235024861 (дата обращения: 11.12.2017).

9. Киселев А. 3. Теория радиолокационного обнаружения на основе использования векторов рассеяния целей. М.: Радио и связь, 2002. 272 с.
10. Поздняк С. И., Мелитицкий В. А. Введение в статистическую теорию поляризации радиоволн. М.: Сов. радио, 1974. 480 с.

11. Павлов В. С., Турнецкая Е. Л. Синтез пространственно-кольцевого пеленгатора источника поляризованного радиоизлучения // Информационно-управляющие системы. 2014. № 6. С. 6-12.

12. Гоноровский И. С. Радиотехнические цепи и сигналы. М.: Дрофа, 2006. 720 с.

13. Справочник по специальным функциям / под ред. М. Абрамовица и И. Стиган. М.: Наука, 1979. 832 с.

14. Бартон Д., Вард Г. Справочник по радиолокационным измерениям. М.: Сов. радио, 1976. 392 с.

Статья поступила в редакцию 15 декабря 2017 г.

Блудов Александр Александрович - доктор технических наук (1996), профессор (1998), главный конструктор АО «НПП "Радар ММС"». Автор более 150 научных работ. Сфера научных интересов - радиолокационные и радионавигационные информационно-управляющие системы.

E-mail: b-sansan@yandex.ru

Горбатовский Глеб Александрович - аспирант и инженер АО «НПП "Радар ММС"», окончил СанктПетербурский политехнический университет им. Петра Великого (2016) по специальности "Техническая физика". Автор шести научных публикаций. Сфера научных интересов - радиолокация; цифровая обработка сигналов.

E-mail: gorbatovsky.g@gmail.com

Павлов Владислав Станиславович - доктор технических наук (2013), профессор (2014) кафедры проблемно-ориентированных вычислительных комплексов Санкт-Петербургского государственного университета аэрокосмического приборостроения, ведущий научный сотрудник АО «НПП "Радар ММС"». Автор более 160 научных работ. Сфера научных интересов - пространственно-временная обработка сигналов в информационно-управляющих системах.

E-mail:w14z@yandex.ru

Суворов Александр Фёдорович - кандидат технических наук (1987), доцент (1989). Начальник лаборатории АО «НПП "Радар ММС"». Автор более 140 научных работ. Сфера научных интересов - системный анализ и управление, радиолокационные системы наведения.

E-mail: vorovus52@yandex.ru

\section{REFERENCES}

1. Skolnik M. Vvedenie $v$ tekhniku radiolokatsionnykh system [Introduction to Radar System Technology]. Moscow, Mir, 1965, 748 p. (In Russian)

2. Gutkin L. S. Printsipy radioupravleniya bespilotnymi objektami [Principles of Unmanned Object Radio Control]. Moscow, Sov. radio, 1959, 384 p. (In Russian)

3. Indenbom M. V. Antennye reshetki podvizhnykh obzornykh RLS. Teoriya, raschet, konstruktsii [Antenna Arrays of Mobile Surveillance Radar. Theory, Calculation, Design]. Moscow, Izd-vo "Radiotekhnika", 2015, 416 p. (In Russian)

4. Bludov A. A., Gorbatovskii G. A., Pavlov V. S., Suvorov A. F. The Method of Two-Plane Wide-Angle Direction Finding of Radio Emission Source. XIX Vserossiiskaya nauchno-prakticheskaya konferentsiya "Aktual'nye problemy zashchity i bezopasnosti" [XIX All-Russia Research and Practice Conference "Security and Safety Actual Issues"]. SPb, NPO SM-RARAN, 2016, vol. 4, pp. 468-472. (In Russian)

5. Kuz'min S. Z. Tsifrovaya radiolokatsiya. Vvedenie $v$ teoriyu [Digital Radar. Introduction to Theory]. Kiev, KVITs, 2000, 428 p. (In Russian)

6. Kozlov A. I., Logvin A. I, Sarychev V. A. Polyarizatsiya radiovoln: $v 3$ kn. Kn. 2: Radiolokatsionnaya polyarimetriya [Polarization of Radio Waves: In 3 Books. Book. 2: Radar-Polar Polarimetry]. Moscow, Radiotekhnika, 2007, 640 p. (In Russian)
7. Kanareikin D. B., Potekhin V. A., Shishkin I. F. Morskaya polyarimetriya [Marine Polarimetry]. Leningrad, Sudostroenie, 1968, 328 p. (In Russian)

8. A Variability Study of Ka-Band HRR Polarimetric Signatures on Eleven T-72 Tanks. Available at: https://www.re-searchgate.net/publication/235024861 (accessed: 11 De-cember 2017).

9. Kiselev A. Z. Teoriya radiolokatsionnogo obnaruzheniya na osnove ispol'zovaniya vektorov rasseyaniya tselei [Theory of Radar Detection Based on Target Scattering Vectors]. Moscow, Radio i svyaz', 2002, 272 p. (In Russian)

10. Pozdnyak S. I., Melititskii V. A. Vvedenie v statisticheskuyu teoriyu polyarizatsii radiovoln [Introduction to Radio Wave Polarization Statistic Theory]. Moscow, Sov. radio, 1974, 480 p. (In Russian)

11. Pavlov V. S., Turnetskaya E. L. Synthesis of the Space-Ring Direction Finder of the Source of Polarized Radio Emission. Informatsionno-upravlyayushchie sistemy [Information and Control Systems]. 2014, no. 6, pp. 6-12. (In Russian)

12. Gonorovskii I. S. Radiotekhnicheskie tsepi i signaly [Radio Circuits and Signals]. Moscow, Izd-vo "Drofa", 2006, 720 p. (In Russian) 
13. Handbook of Mathematical Functions With Formulas, Graphs, and Mathematical Tables, ed. by M. Abramowitz, I. A. Stegun. Dover, 1964, 1046 p.

Received December, 15, 2017

Alexandr A. Bludov - D.Sc. in Engineering (1996), Professor (1998), chief designer of Research and Development Enterprise JSC "Radar MMS". The author of more than 150 scientific publications. Area of expertise: control systems in radar and radio navigation applications.

E-mail: b-sansan@yandex.ru

Gleb A. Gorbatovskiy - Master's Degree in Technical Physics in Peter the Great St. Petersburg Polytechnical University (2016). Postgraduate student and engineer at Research and Development Enterprise JSC "Radar MMS". The author of 6 scientific publications. Area of expertise: radiolocation, digital signal processing. E-mail: gorbatovsky.g@gmail.com

Vladislav S. Pavlov - D.Sc. in Engineering (2013), Professor (2014) of the Department of Major Problem Oriented Computer Complexes at St. Petersburg State University of Aerospace Instrumentation, leading researcher at Research and Development Enterprise JSC "Radar MMS". The author of more than 160 scientific publications. Area of expertise: temporal and spatial signal processing in control systems.

E-mail: w14z@yandex.ru

Aleksandr F. Suvorov - Ph.D. in Engineering (1987), Associate Professor (1989). Head of laboratory at JSC "Radar MMS". Author of more than 140 scientific publications. Area of expertise: system analysis and design, radar navigation systems.

E-mail: vorovus52@yandex.ru 